\title{
Full-State Linearization and Stabilization of SISO Markovian Jump Nonlinear Systems
}

\author{
Zhongwei Lin, Jizhen Liu, and Yuguang Niu \\ State Key Laboratory of Alternate Electrical Power System with Renewable Energy Sources, School of Control and Computer \\ Engineering, North China Electric Power University, Beijing 102206, China
}

Correspondence should be addressed to Zhongwei Lin; linzhongwei2003@tom.com

Received 18 January 2013; Revised 13 March 2013; Accepted 18 March 2013

Academic Editor: Rongni Yang

Copyright (C) 2013 Zhongwei Lin et al. This is an open access article distributed under the Creative Commons Attribution License, which permits unrestricted use, distribution, and reproduction in any medium, provided the original work is properly cited.

This paper investigates the linearization and stabilizing control design problems for a class of SISO Markovian jump nonlinear systems. According to the proposed relative degree set definition, the system can be transformed into the canonical form through the appropriate coordinate changes followed with the Markovian switchings; that is, the system can be full-state linearized in every jump mode with respect to the relative degree set $\{n, \ldots, n\}$. Then, a stabilizing control is designed through applying the backstepping technique, which guarantees the asymptotic stability of Markovian jump nonlinear systems. A numerical example is presented to illustrate the effectiveness of our results.

\section{Introduction}

Markovian jump systems have been studied since the pioneering work on quadratic control of linear jump systems in the 1960s. It switches from one mode to another in a random way, and the switching between the modes is governed by a Markov process with discrete and finite state space. Due to the advantage of the probabilistic description through Markov process, Markovian jump systems are well suited to model changes induced by external causes, for example, random faults, unexpected events, and uncontrolled configuration changes; see [1-5]. Noticeable achievements have been made in the past three decades on stability analysis and controller design of linear Markovian jump systems (see [1, 2, 6-11] and the references therein). Reference [7] has investigated the problem of control for discrete time-delay linear systems with Markovian jump parameters. References $[10,11]$ discussed the sliding mode control problems for Markovian jump linear singular systems. Meanwhile, many authors have considered the results for linear Markovian jump time-delay systems; see $[7,12-15]$ and the references therein.

On the other hand, many authors have turned to study the stability and stabilization problems of Markovian jump nonlinear systems. Reference [16] especially, has established the framework of stability analysis for Markovian jump nonlinear system. Reference [17] has proposed the Kalman-Yacubovitch-Popov (KYP) Lemma for the passivity of Markovian jump nonlinear system. [18] discussed the $H_{\infty}$ control design for Markovian jump nonlinear system. Reference [19] has discussed the stabilization problems for interconnected Markovian jump nonlinear system from the viewpoint of dissipativity theory. Reference [20] has discussed the stabilization problem for a class of Markovian jump nonlinear systems in the special canonical form. All these works extended results for Markovian jump nonlinear system from the traditional nonlinear control theory, such as [21-26].

The purpose of this paper is to fill some gaps on the geometric control theory for Markovian jump nonlinear systems. It is well known that the geometric control theory plays an important role in the stabilization of nonlinear systems; see [23, 25, 26]. However, as to the Markovian jump systems, there is still no related research work on this topic. The difficulty concretes on how to transform the Markovian jump nonlinear system into the canonical form? Motivated by this point, this paper investigates the linearization and stabilizing control design problems for Markovian jump nonlinear systems. More concretely, the main contribution of this paper contains the following aspects. 
(i) The definition of relative degree set is proposed for Markovian jump nonlinear systems. Because there exist many nonlinear subsystems corresponding to different jump mode, it would be more appropriate to define the relative degrees of the system in terms of set.

(ii) The full-state linearization method for Markovian jump nonlinear system is proposed. For every subsystem, the corresponding diffeomorphism can be constructed to transform the system into the triangle form, which implies that all the subsystems can be transformed to the common triangle form, but in different coordinates.

(iii) The backstepping technique is applied for the stabilizing control design of the transformed triangle form of Markovian jump nonlinear system.

The rest of this paper is organized as follows. Section 2 discusses the feedback linearization of Markovian jump systems. According to the proposed relative degree set definition, the appropriate coordinate is adopted under which the system can be transformed into the canonical form followed with the Markovian switchings. Section 3 discusses the stabilizing control design through applying the backstepping technique on the canonical form of Markovian jump nonlinear systems. Section 4 presents a numerical example to illustrate the effectiveness of our results. Section 5 concludes this paper.

For convenience, we adopt the following notation: $A^{\prime}$ is the transpose of the corresponding matrix $A . A \geq 0(A>$ 0 ) is the positive semi-definite (positive-definite) matrix. $R^{n}$ is $n$-dimensional Euclidean space. $\|x\|$ is 2 -norm of a vector $x \in R^{n} . \mathscr{K}$ is the family of all increasing functions $k$, such that $k(0)=0, k(t)>0$ for $t>0 . \mathscr{K}_{\infty}$ is the family of all functions $k \in \mathscr{K}$ with $k(\infty)=\infty$.

\section{Feedback Linearization}

Consider the following controlled Markovian jump nonlinear system

$$
\begin{gathered}
\dot{x}=f\left(x, r_{t}\right)+g\left(x, r_{t}\right) u, \\
y=h\left(x, r_{t}\right),
\end{gathered}
$$

in a fixed complete probability space $(\Omega, \mathscr{F}, P)$, where $x(t) \epsilon$ $R^{n}$ is the state vector, $y(t) \in R$ is the controlled output, and $u(t) \in R$ is the control input. $\left\{r_{t}, t \geq 0\right\}$ is the continuous-time Markov process taking values in a finite set $\varphi=\{1,2, \ldots, N\}$, which represents the switching between the different modes and its dynamics are described by the following transitions probabilities:

$$
P\left[r_{t+h}=j \mid r_{t}=i\right]= \begin{cases}\lambda_{i j} h+o(h), & i \neq j, \\ 1+\lambda_{i i} h+o(h), & i=j,\end{cases}
$$

where $\lambda_{i j}$ is the transition rate from mode $i$ to $j$ with $\lambda_{i j} \geq 0$ when $i \neq j$ and $\lambda_{i i}=-\sum_{j=1, j \neq i}^{N} \lambda_{i j}$ and $o(h)$ is such that $\lim _{h \rightarrow 0} o(h) / h=0$. The Markovian switching parameter process is assumed to be completely measurable at any time instant. For simplicity of notations, take $f_{i}$ for $f(x, i)$ when $r_{t}=i \in \varphi$ and so forth. $f_{i}, g_{i}$, and $h_{i}$ are Lipschitz satisfying a linear growth condition, which guarantees that the system (1) has a unique strong solution [1].

Motivated by the nonlinear control theory for the deterministic systems (see $[25,26]$ and the references therein), we present the following definition related to the relative degree for Markovian jump nonlinear systems.

Definition 1 (relative degree set). The Markovian jump nonlinear system (1) is said to have the relative degree set $\left\{\rho_{1}, \rho_{2}, \ldots, \rho_{N}\right\}$, if for every $r_{t}=i \in \varphi$, the corresponding system is with the relative degree $\rho_{i}$, that is,

$$
\begin{gathered}
L_{g_{i}} L_{f_{i}}^{j-1} h_{i}(x)=0, \\
L_{g_{i}} L_{f_{i}}^{\rho_{i}-1} h_{i}(x) \neq 0,
\end{gathered}
$$

where $j=1,2, \ldots, \rho_{i}-1$.

Obviously, the above definition is a generalization of the relative degree definition in $[25,26]$. Here, the relative degree set contains all the relative degree, of all the subsystem for every system mode $i \in \varphi$, which is determined by the characteristic of Markovian jump nonlinear systems.

Note that we start off with the case when all the subsystems are SISO, because it gives more insight into computations and helps understanding the concepts which can be generalized to multivariable systems. Moreover, we consider the system (1) with the relative degree set $\{n, \ldots, n\}$, which satisfies the following condition according to Definition 1:

$$
\begin{aligned}
& L_{g_{i}} L_{f_{i}}^{n-1} h_{i}(x)=0, \\
& L_{g_{i}} L_{f_{i}}^{n-1} h_{i}(x) \neq 0 .
\end{aligned}
$$

Now, let us consider a set of new coordinates

$$
\xi^{(i)}=\phi_{i}(x), \quad i \in \varphi,
$$

which indeed stands for the following coordinates:

$$
\xi^{(i)}=\phi_{i}(x)=\left[\begin{array}{c}
\phi_{i 1}(x) \\
\phi_{i 2}(x) \\
\vdots \\
\phi_{i n}(x)
\end{array}\right]=\left[\begin{array}{c}
h_{i}(x) \\
L_{f_{i}}^{1} h_{i}(x) \\
\vdots \\
L_{f_{i}}^{n-1} h_{i}(x)
\end{array}\right]
$$

Take the new coordinate variables as follows:

$$
\begin{gathered}
y_{1}^{(i)}=h_{i}(x), \\
y_{2}^{(i)}=L_{f_{i}}^{1} h_{i}(x),
\end{gathered}
$$

$$
y_{n}^{(i)}=L_{f_{i}}^{n-1} h_{i}(x) .
$$


For every $i \in \varphi$, if the above $\phi_{i}$ and its inverse $\phi_{i}^{-1}$ are both smooth functions, $\phi_{i}$ is called a diffeomorphism; see $[25,26]$. Under this assumption, we have

$$
\begin{gathered}
\dot{y}_{1}^{(i)}=y_{2}^{(i)}, \\
\dot{y}_{2}^{(i)}=y_{3}^{(i)}, \\
\vdots \\
\dot{y}_{n}^{(i)}=L_{f_{i}}^{n} h_{i}(x)+L_{g_{i}} L_{f_{i}}^{n-1} h_{i}(x) u .
\end{gathered}
$$

Due to the fact that $L_{g_{i}} L_{f_{i}}^{n-1} h_{i}(x) \neq 0$, we can set the control law as

$$
u=\left(L_{g_{i}} L_{f_{i}}^{n-1} h_{i}(x)\right)^{-1}\left(v-L_{f_{i}}^{n} h_{i}(x)\right),
$$

then the system (8) is feedback equivalent to the following linearized form:

$$
\begin{gathered}
\dot{\xi}^{(i)}=A \xi^{(i)}+B v, \\
y=C \xi^{(i)}
\end{gathered}
$$

where

$$
\begin{gathered}
A=\left[\begin{array}{ccccc}
0 & 1 & 0 & \cdots & 0 \\
0 & 0 & 1 & \cdots & 0 \\
\vdots & \ddots & & \vdots \\
\vdots & & 0 & 1 \\
0 & \cdots & 0 & 0
\end{array}\right]_{n \times n}, \quad B=\left[\begin{array}{c}
0 \\
0 \\
\vdots \\
0 \\
1
\end{array}\right]_{n \times 1}, \\
C=\left[\begin{array}{lllll}
1 & 0 & \cdots & 0 & 0
\end{array}\right]_{1 \times n},
\end{gathered}
$$

which can be regarded as the canonical form for Markovian jump nonlinear system.

Through the above discussion, we have transformed the Markovian jump system into the triangle form corresponding to different $\xi^{(i)}$-coordinate, respectively. Hence, we have established the relationship between the proposed relative degree set definition and the full-state linearization for Markovian jump nonlinear systems as follows.

Theorem 2. If the system (1) has a relative set $\{n, \ldots, n\}$, then the system is full-state feedback linearizable.

Remark 3. The above result can be regarded as a generalized work from deterministic nonlinear control theory to Markovian jump nonlinear system. The key point concentrates on taking the appropriate coordinate changes followed with the Markovian switchings. In this way, we can transform the subsystems into the canonical form (10), which have the same triangle structure but within different coordinates.

Following with the update of the Markovian jump mode $r_{t}=i$, we can transform the $x$-system into the corresponding $\xi^{(i)}$-system, which also works for the stability conditions. Considering the system (1), for any nonnegative functions
$U(x, i) \in C\left(R^{n}\right): R^{n} \rightarrow R^{+}$, with $U(0, i)=0, i \in \varphi$, we have

$$
\mathscr{L} U(x, i)=\frac{\partial U^{\prime}(x, i)}{\partial x} f_{i}+\sum_{j=1}^{N} \lambda_{i j} U(x, j),
$$

where $\mathscr{L}$ is the infinitesimal generator of the system; see [1, 17]. Through the coordinate transformation, denoting and

$$
\begin{gathered}
\widehat{V}\left(\xi^{(i)}, i\right)=V\left(\phi_{i}(x), i\right)=U(x, i), \\
\widehat{V}\left(\xi^{(i)}, j\right)=V\left(\xi^{(j)}, j\right)
\end{gathered}
$$

we have

$$
\begin{aligned}
& \mathscr{L} V\left(\xi^{(i)}, i\right) \\
&= \mathscr{L} U\left(x^{(i)}, i\right)=\left(\frac{\partial U^{\prime}(x, i)}{\partial x} f_{i}+\sum_{j=1}^{N} \lambda_{i j} U(x, j)\right) \\
&=\left(\frac{\partial U^{\prime}(x, i)}{\partial x} f_{i}+\lambda_{i 1} U(x, 1)+\cdots+\lambda_{i N} U(x, N)\right) \\
&= \frac{\partial V^{\prime}\left(\xi^{(i)}, i\right)}{\partial \xi^{(i)}} A+\lambda_{i 1} V\left(\xi^{(1)}, 1\right) \\
&+\cdots+\lambda_{i N} V\left(\xi^{(N)}, N\right) \\
&= \frac{\partial V^{\prime}\left(\xi^{(i)}, i\right)}{\partial \xi^{(i)}} A+\lambda_{i 1} \widehat{V}\left(\xi^{(i)}, 1\right) \\
&+\cdots+\lambda_{i N} \widehat{V}\left(\xi^{(i)}, N\right) \\
&= \frac{\partial V^{\prime}\left(\xi^{(i)}, i\right)}{\partial \xi^{(i)}} A+\sum_{j=1}^{N} \lambda_{i j} \widehat{V}\left(\xi^{(i)}, j\right) .
\end{aligned}
$$

Due to the fact that the stability of the system (1) is equivalent to the system (10), the stability condition for the system (1) in [1] can be transformed with respect to the system (10) as follows.

Lemma 4. The unforced system (10) is globally asymptotically stable in probability (GASP), if there is a set of positive-definite functions $V\left(\xi^{(i)}, i\right) \in C\left(R^{n}\right)$ with $V\left(\xi^{(i)}, i\right) \rightarrow \infty$ as $\left\|\xi^{(i)}\right\| \rightarrow$ $\infty, c \in \mathscr{K}, i \in \varphi$, such that

$$
\begin{aligned}
\mathscr{L} V\left(\xi^{(i)}, i\right) & =\frac{\partial V^{\prime}\left(\xi^{(i)}, i\right)}{\partial \xi^{(i)}} A+\sum_{j=1}^{N} \lambda_{i j} \widehat{V}\left(\xi^{(i)}, j\right) \\
& \leq-c\left(\left\|\xi^{(i)}\right\|\right) .
\end{aligned}
$$

Proof. According to Definition 5.34 and Theorem 5.36 of [16], the asymptotical stability of the system can be guaranteed by

$$
\frac{\partial V^{\prime}\left(\xi^{(i)}, i\right)}{\partial \xi^{(i)}} A+\sum_{j=1}^{N} \lambda_{i j} V\left(\xi^{(j)}, j\right) \leq-c\left(\left\|\xi^{(i)}\right\|\right) .
$$


Then, considering the transformation of (14), the above condition can be represented in the form of (16). The proof of Lemma 4 is completed.

Obviously, the above results also hold for the closedloop system under the control law. Although with the relative degree set $\{n, \ldots, n\}$, the Markovian jump nonlinear system (1) can be feedback equivalent to the triangle form (10), but how to construct the control $v$ such that the $\xi^{(i)}$-system can be stabilized following with the Markovian switchings, which is an interesting problem and will be discussed in the next section.

\section{Stabilizing Control Design}

In this section, we consider the backstepping control design of the Markovian jump nonlinear system (1). Through the discussion in Section 2, the system (1) can be transformed into the following $\xi^{(i)}$-system:

$$
\begin{gathered}
\dot{y}_{1}^{(i)}=y_{2}^{(i)}, \\
\dot{y}_{2}^{(i)}=y_{3}^{(i)}, \\
\vdots \\
\dot{y}_{n}^{(i)}=v .
\end{gathered}
$$

Now, we continue to take the following coordinate change:

$$
\begin{gathered}
z_{1}^{(i)}=y_{1}^{(i)}, \\
z_{2}^{(i)}=y_{2}^{(i)}-c_{1}^{(i)}, \\
\vdots \\
z_{n}^{(i)}=y_{n}^{(i)}-c_{n-1}^{(i)},
\end{gathered}
$$

where the known function $c_{k}^{(i)}$ is smooth and satisfying $c_{k}^{(i)}(0)=0, k \in\{1, \ldots, n-1\}, i \in \varphi$. Equivalently, we have

$$
\begin{gathered}
y_{1}^{(i)}=z_{1}^{(i)}, \\
y_{2}^{(i)}=z_{2}^{(i)}+c_{1}^{(i)}, \\
\vdots \\
y_{n}^{(i)}=z_{n}^{(i)}+c_{n-1}^{(i)} .
\end{gathered}
$$

Furthermore, for every $i \in \varphi$, between any two of the following coordinates

$$
\left[\begin{array}{c}
z_{1}^{(i)} \\
\vdots \\
z_{n}^{(i)}
\end{array}\right] \Longleftrightarrow\left[\begin{array}{c}
y_{1}^{(i)} \\
\vdots \\
y_{n}^{(i)}
\end{array}\right] \Longleftrightarrow\left[\begin{array}{c}
x_{1} \\
\vdots \\
x_{n}
\end{array}\right] \Longleftrightarrow\left[\begin{array}{c}
y_{1}^{(j)} \\
\vdots \\
y_{n}^{(j)}
\end{array}\right] \Longleftrightarrow\left[\begin{array}{c}
z_{j}^{(i)} \\
\vdots \\
z_{n}^{(j)}
\end{array}\right]
$$

or

$$
Z_{n}^{(i)} \Longleftrightarrow \xi^{(i)} \Longleftrightarrow x \Longleftrightarrow \xi^{(j)} \Longleftrightarrow Z_{n}^{(j)},
$$

it can be found that there exists the corresponding inverse transformation, which is also a diffeomorphism. For simplicity of notations, take $Z_{n}^{(i)}=T_{i}(x)$, then we have

$$
Z_{n}^{(j)}=T_{j}\left(T_{i}^{-1}\left(Z_{n}^{(i)}\right)\right)
$$

Denote

$$
\begin{aligned}
\xi_{k}^{(i)} & =\left[y_{1}^{(i)}, \ldots, y_{k}^{(i)}\right]^{\prime}, \\
Z_{k}^{(i)} & =\left[z_{1}^{(i)}, \ldots, z_{k}^{(i)}\right]^{\prime}, \\
F_{k}^{(i)} & =\left[y_{2}^{(i)}, \ldots, y_{k-1}^{(i)}\right]^{\prime} .
\end{aligned}
$$

As follows, we discuss the control design for the system (18). Firstly, for every $i \in \varphi$, taking the following set of Lyapunov functions

$$
\begin{aligned}
V\left(\xi^{(i)}, i\right) & =\frac{1}{2} \sum_{k=1}^{n}\left(y_{k}^{(i)}-c_{k-1}^{(i)}\right)^{2} \\
& =\frac{1}{2} \sum_{k=1}^{n}\left(z_{k}^{(i)}\right)^{2} .
\end{aligned}
$$

Motivated by (16), the infinite small operator for the system (18) is given as follows:

$$
\begin{aligned}
\mathscr{L} V\left(\xi^{(i)}, i\right)= & y_{1}^{(i)} y_{2}^{(i)} \\
& +\sum_{k=2}^{n-1}\left(y_{k}^{(i)}-c_{k-1}^{(i)}\right)\left(y_{k+1}^{(i)}-\frac{\partial c_{k-1}^{(i)} \dot{\xi}_{k-1}^{(i)}}{\partial \xi_{k-1}^{(i)}}\right) \\
& +\left(y_{n}^{(i)}-c_{n-1}^{(i)}\right)\left(v-\frac{\partial c_{n-1}^{(i)}}{\partial \xi_{n-1}^{(i)}} \dot{\xi}_{n-1}^{(i)}\right) \\
& +\frac{1}{2} \sum_{j=1}^{N} \sum_{k=1}^{n} \lambda_{i j}\left(y_{k}^{(j)}-c_{k-1}^{(i)}\right)^{2} \\
= & z_{1}^{(i)}\left(z_{2}^{(i)}+c_{1}^{(i)}\right) \\
& +\sum_{k=2}^{n-1} z_{k}^{(i)}\left(z_{k+1}^{(i)}+c_{k}^{(i)}-\frac{\partial c_{k-1}^{(i)}}{\partial \xi_{k-1}^{(i)}} F_{k-1}^{(i)}\right) \\
& +z_{n}^{(i)}\left(v-\frac{\partial c_{n-1}^{(i)}}{\partial \xi_{n-1}^{(i)}} F_{n-1}^{(i)}\right) \\
& +\frac{1}{2} \sum_{j=1}^{N} \lambda_{i j}\left(\sum_{k=1}^{n}\left(z_{k}^{(j)}\right)^{2}\right) .
\end{aligned}
$$


Using Young's inequality, we have

$$
\begin{aligned}
\sum_{k=1}^{n-1} z_{k}^{(i)} z_{k+1}^{(i)} & \leq \sum_{k=1}^{n-1}\left(\frac{1}{2} \beta_{k}^{2}\left(z_{k}^{(i)}\right)^{2}+\frac{1}{\left(2 \beta_{k}^{2}\right)\left(z_{k+1}^{(i)}\right)^{2}}\right) \\
& =\sum_{k=1}^{n}\left(\frac{1}{2} \beta_{k}^{2}+\frac{1}{2 \beta_{k-1}^{2}}\right)\left(z_{k}^{(i)}\right)^{2},
\end{aligned}
$$

where $\beta_{k}>0$ are designed parameters. Then, (26) reduces to

$$
\begin{aligned}
\mathscr{L} V\left(\xi^{(i)}, i\right) \leq & z_{1}^{(i)}\left[\left(\frac{1}{2} \beta_{1}^{2}+\frac{1}{2 \beta_{0}^{2}}\right) z_{1}^{(i)}+c_{1}^{(i)}\right] \\
& +\sum_{k=2}^{n-1} z_{k}^{(i)}\left[\left(\frac{1}{2} \beta_{k}^{2}+\frac{1}{2 \beta_{k-1}^{2}}\right) z_{k}^{(i)}\right. \\
& \left.+c_{k}^{(i)} L-\frac{\partial c_{k-1}^{(i)}}{\partial \xi_{k-1}^{(i)}} F_{k-1}^{(i)}\right] \\
& +\left(z_{n}^{(i)}\right)\left(v-\frac{\partial c_{k-1}^{(i)}}{\partial \xi_{k-1}^{(i)}} F_{n-1}^{(i)}\right) \\
& +\sum_{j=1}^{N} \lambda_{i j}\left(\frac{1}{2} \sum_{k=1}^{n}\left(z_{k}^{(j)}\right)^{2}\right) .
\end{aligned}
$$

Taking

$$
\begin{gathered}
c_{1}^{(i)}=-\alpha_{1} z_{1}^{(i)}-\left(\frac{1}{2} \beta_{1}^{2}+\frac{1}{2 \beta_{0}^{2}}\right) z_{1}^{(i)}, \\
c_{k}^{(i)}=\alpha_{k} z_{k}^{(i)}-\left(\frac{1}{2} \beta_{k}^{2}+\frac{1}{2 \beta_{k-1}^{2}}\right) z_{k}^{(i)}+\frac{\partial c_{k-1}^{(i)}}{\partial \xi_{k-1}^{(i)}} F_{k-1}^{(i)},
\end{gathered}
$$

where $\alpha_{k}>0$ are also designed parameters. Note that, the final value of the designed control $v$ can be regulated through adjusting the parameters $\alpha_{k}$ and $\beta_{k}$, which can benefit many practical control design requirements. With fixed appropriate $\alpha_{k}$ and $\beta_{k}$, the transformation of (19) and (23) can be constructed directly.

Considering the last term of (29), through the transformation (22), we have

$$
\widehat{V}\left(\xi^{(i)}, j\right)=\widehat{V}_{n}\left(Z_{n}^{(i)}, j\right)=\left.\frac{1}{2} \sum_{k=1}^{n}\left(z_{k}^{(j)}\right)^{2}\right|_{z_{n}^{(i)}=T_{j}\left(T_{i}^{-1}\left(Z_{n}^{(i)}\right)\right)} .
$$

\section{Denote}

$$
\begin{aligned}
\widehat{V}_{n-1}\left(Z_{n-1}^{(i)}, j\right) & =\widehat{V}_{n}\left(Z_{n-1}^{(i)}, 0, j\right), \Delta \widehat{V}_{n}\left(Z_{n}^{(i)}, j\right) \\
& =\left.\int_{0}^{1} \frac{\partial \widehat{V}_{n}\left(Z_{n-1}^{(i)}, \varsigma^{(i)}, j\right)}{\partial \varsigma^{(i)}}\right|_{\varsigma^{(i)}=\tau z_{n}^{(i)}} d \tau,
\end{aligned}
$$

then we have

$$
\widehat{V}_{n}\left(Z_{n}^{(i)}, j\right)=\widehat{V}_{n-1}\left(Z_{n-1}^{(i)}, j\right)+z_{n}^{(i)} \Delta \widehat{V}_{n}\left(Z_{n}^{(i)}, j\right) .
$$

Moreover, repeating this procedure, we have

$$
\begin{aligned}
\widehat{\mathrm{V}}_{n}\left(Z_{n}^{(i)}, j\right)= & \left.\frac{1}{2}\left(\sum_{k=1}^{n}\left(z_{k}^{(j)}\right)^{2}\right)\right|_{Z_{n}^{(j)}=T_{j}\left(T_{i}^{-1}\left(Z_{n}^{(i)}\right)\right)} \\
= & \widehat{V}_{n-1}\left(Z_{n-1}^{(i)}, j\right)+z_{n}^{(i)} \Delta \widehat{V}_{n}\left(Z_{n}^{(i)}, j\right) \\
& \vdots \\
= & \widehat{V}_{1}\left(Z_{1}^{(i)}, j\right)+\sum_{k=2}^{n} z_{k}^{(i)} \Delta \widehat{V}_{k}\left(Z_{k}^{(i)}, j\right) .
\end{aligned}
$$

Note that

$$
\begin{aligned}
\widehat{V}_{1}\left(Z_{1}^{(i)}, j\right) & =\widehat{V}\left(z_{1}^{(i)}, 0, \ldots, 0, j\right) \\
& =\widehat{V}(0, \ldots, 0, j)+z_{1}^{(i)} \Delta \widehat{V}_{1}=z_{1}^{(i)} \Delta \widehat{V}_{1} .
\end{aligned}
$$

Finally, we have

$$
\widehat{V}\left(\xi^{(i)}, j\right)=\sum_{k=1}^{n} z_{k}^{(i)} \Delta \widehat{V}_{k}\left(Z_{k}^{(i)}, j\right) .
$$

Through the above discussion on the coupled term, we can have

$$
\begin{aligned}
\mathscr{L} V\left(\xi^{(i)}, i\right) \leq & z_{1}^{(i)}\left[\left(\frac{1}{2} \beta_{1}^{2}+\frac{1}{2 \beta_{0}^{2}}\right) z_{1}^{(i)}+c_{1}^{(i)}\right] \\
& +\sum_{k=2}^{n-1} z_{k}^{(i)}\left[\left(\frac{1}{2} \beta_{k}^{2}+\frac{1}{2 \beta_{k-1}^{2}}\right) z_{k}^{(i)}\right. \\
& +\left(z_{n}^{(i)}\right)\left(v-\frac{\partial c_{n-1}^{(i)}}{\partial \xi_{n-1}^{(i)}} F_{n-1}^{(i)}\right) \\
& +\sum_{j=1}^{N} \lambda_{i j}\left(\sum_{k=1}^{n} z_{k}^{(i)} \Delta \widehat{V}_{k}^{(i)}\left(Z_{k}^{(i)}, j\right)\right) \\
+ & z_{1}^{(i)}\left[\left(\frac{1}{\partial \xi_{k-1}^{(i)}} F_{k-1}^{(i)}\right]\right. \\
& +\left(z_{n}^{(i)}\right)\left(v-\frac{\partial c_{n-1}^{(i)}}{\partial \xi_{n-1}^{(i)}} F_{n-1}^{(i)}+\sum_{j=1}^{N} \lambda_{i j} \Delta \widehat{V}_{n}\left(Z_{n}^{(i)}, j\right)\right. \\
& +\sum_{k=1}^{n-1} z_{k}^{(i)}\left[\left(\frac{1}{2} \beta_{k}^{2}+\frac{1}{2 \beta_{k-1}^{2}}\right) z_{k}^{(i)}+c_{k}^{(i)}\right. \\
& \left.+\sum_{j=1}^{N} \lambda_{i j} \Delta \widehat{V}_{1}\left(Z_{1}^{(i)}, j\right)+c_{1}^{(i)}\right] \\
& \left.-\frac{\partial c_{k-1}}{\partial \xi_{k-1}^{(i)}} F_{k-1}^{(i)}+\sum_{j=1}^{N} \lambda_{i j} \Delta \widehat{V}_{k}\left(Z_{k}^{(i)}, j\right)\right]
\end{aligned}
$$


Through updating

$$
\begin{aligned}
c_{1}^{*(i)}= & -\alpha_{1} z_{1}^{(i)}-\left(\frac{1}{2} \beta_{1}^{2}+\frac{1}{2 \beta_{0}^{2}}\right) z_{1}^{(i)} \\
& -\sum_{j=1}^{N} \lambda_{i j} \Delta \widehat{V}_{1}\left(Z_{1}^{(i)}, j\right), \\
c_{k}^{*(i)}= & -\alpha_{k} z_{k}^{(i)}-\left(\frac{1}{2} \beta_{k}^{2}+\frac{1}{2 \beta_{k-1}^{2}}\right) z_{k}^{(i)} \\
& +\frac{\partial c_{k-1}^{(i)}}{\partial \xi_{k-1}^{(i)}} F_{k-1}^{(i)}-\sum_{j=1}^{N} \lambda_{i j} \Delta \widehat{V}_{k}\left(Z_{k}^{(i)}, j\right),
\end{aligned}
$$

we can get the control as follows:

$$
v^{*}=-\alpha_{n} z_{n}^{(i)}+\frac{\partial c_{n-1}^{*(i)}}{\partial \xi_{n-1}^{(i)}} F_{n-1}^{(i)}-\sum_{j=1}^{N} \lambda_{i j} \Delta \widehat{V}_{n}\left(Z_{n}^{(i)}, j\right) .
$$

Moreover, by (9), we can design the original control $u$ as follows:

$$
u^{*}=\left(L_{g_{i}} L_{f_{i}}^{n-1} h_{i}(x)\right)^{-1}\left(v^{*}-L_{f_{i}}^{n} h_{i}(x)\right)
$$

Combined with the above discussion, we get to the following theorem.

Theorem 5. If the system (1) has a relative set $\{n, \ldots, n\}$, then the closed-loop system under the control $u^{*}$ is GASP.

Proof. Based on Lemma 4 and (37), sufficient condition (16) for the closed-loop system reduces to

$$
\mathscr{L} V\left(\xi^{(i)}, i\right) \leq-\sum_{k=1}^{n} \alpha_{k}\left(z_{k}^{(i)}\right)^{2} \leq-c V\left(\xi^{(i)}, i\right),
$$

where $c=2 \min \left\{\alpha_{1}, \alpha_{2}, \ldots, \alpha_{n}\right\}$. According to Theorem 5.37 in [16], for every $i \in \varphi$, the function $c V\left(\xi^{(i)}, i\right)=(c / 2)\left\|\xi^{(i)}\right\|^{2}$ belongs to $\mathscr{K}$-function, then (42) indicates the system (1) to be GASP. The proof of this theorem is completed.

Remark 6. Obviously, the difficulty of applying the backstepping technique on Markovian jump nonlinear system is that the coupled term in the Lyapunov inequality (16) is determined by the characteristic of the system. However, this work has provided the above line to settle this problem. Future work could be concentrated on reducing the computing complexity, which is mainly caused by the following two aspects. Firstly, we need to check the relative degree set of the system through Definition 1 . Secondly, we need to calculate the backstepping coefficients $c_{i}$ containing the terms $\Delta \widehat{V}_{k}\left(Z_{k}^{(i)}, j\right)$ caused by the additional term (16). Both show the complexity of geometric control theory of Markovian jump nonlinear systems.

Remark 7. The above results only consider the case of Markovian jump nonlinear system with the relative degree set $\{n, \ldots, n\}$. Indeed, we have discussed the full-state linearization problem for every subsystem corresponding to the jump mode $r_{t}=i$, respectively. However, if the subsystem has arbitrary relative degree set, that is, $\left\{\rho_{1}, \rho_{2}, \ldots, \rho_{N}\right\}$, the Markovian jump nonlinear system cannot be full-state linearized, and we need to study the zero-output dynamics of the system. Hence, how to solve the input-output linearization and stabilization problems is very interesting and requires further studies.

\section{Simulation Results}

In this section, we present a numerical example for the nonlinear Markovian jump system (1). Let $r(t)$ be a rightcontinuous Markov chain taking values in $\varphi=\{1,2\}$ with the generator

$$
\Pi=\left(\lambda_{i j}\right)=\left[\begin{array}{cc}
-1 & 1 \\
2 & -2
\end{array}\right] .
$$

Consider the following Markovian jump nonlinear system:

$$
\begin{aligned}
& \text { Mode1: }\left\{\begin{array}{l}
\dot{x}=\left[\begin{array}{c}
-2 x_{1}^{2}+2 x_{2} \\
x_{2}
\end{array}\right]+\left[\begin{array}{l}
0 \\
1
\end{array}\right] u, \\
y=x_{1},
\end{array}\right. \\
& \text { Mode2: }\left\{\begin{array}{l}
\dot{x}=\left[\begin{array}{c}
x_{2}^{2} \\
x_{1}-x_{2}
\end{array}\right]+\left[\begin{array}{l}
1 \\
0
\end{array}\right] u, \\
y=x_{2} .
\end{array}\right.
\end{aligned}
$$

Firstly, based on Definition 1, we discuss the relative set of the above system.

$$
\begin{gathered}
L_{g_{1}} h_{1}=\frac{\partial h_{1}}{\partial x} g_{1}=\left[\begin{array}{ll}
1 & 0
\end{array}\right]\left[\begin{array}{l}
0 \\
1
\end{array}\right]=0, \\
L_{g_{1} L_{f_{1}} h_{1}=} \frac{\partial\left(\left(\partial h_{1} / \partial x\right) f_{1}\right)}{\partial x} g_{1}=\frac{\partial\left(-2 x_{1}^{2}+2 x_{2}\right)}{\partial x} g_{1} \\
=\left[\begin{array}{ll}
-4 x_{1} & 2
\end{array}\right]\left[\begin{array}{l}
0 \\
1
\end{array}\right]=2 \neq 0, \\
L_{g_{2}} h_{2}=\frac{\partial h_{2}}{\partial x} g_{2}=\left[\begin{array}{ll}
0 & 1
\end{array}\right]\left[\begin{array}{l}
1 \\
0
\end{array}\right]=0, \\
L_{g_{2} L_{f_{2}} h_{2}}=\frac{\partial\left(\left(\partial h_{2} / \partial x\right) f_{2}\right)}{\partial x} g_{2}=\frac{\partial\left(x_{1}-x_{2}\right)}{\partial x} g_{2} \\
=\left[\begin{array}{ll}
1 & -1
\end{array}\right]\left[\begin{array}{l}
1 \\
0
\end{array}\right]=1 \neq 0 .
\end{gathered}
$$

Obviously, the above conditions satisfy the assumption in Definition 1, that is, the relative degree set is $\{2,2\}$, which implies that system (44) and (45) can be, respectively, transformed into the following canonical form:

$$
\begin{gathered}
\dot{y}_{1}^{(1)}=y_{2}^{(1)}, \\
\dot{y}_{2}^{(1)}=v, \\
\dot{y}_{1}^{(2)}=y_{2}^{(2)}, \\
\dot{y}_{2}^{(2)}=v .
\end{gathered}
$$




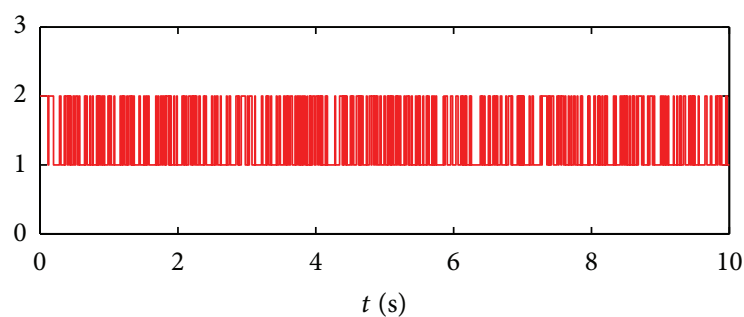

FIGURE 1: State of Markov process $r_{t}$.

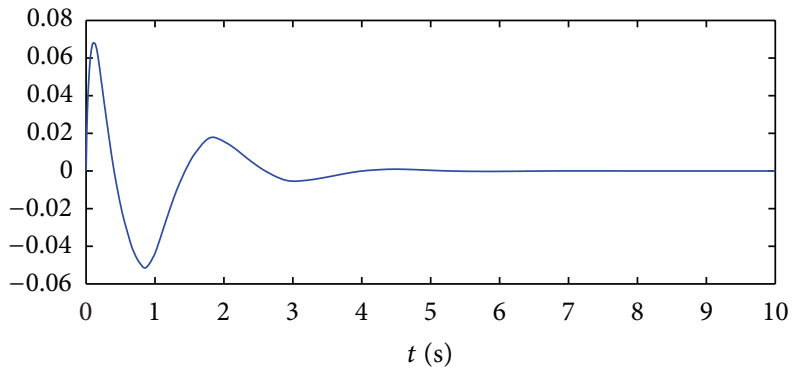

- Output of $y_{1}^{(i)}$

FIgURE 2: Trajectories of state $y_{1}^{(i)}$.

From (29), taking $\alpha_{1}=1, \alpha_{2}=1, \beta_{0}=1$, and $\beta_{1}=1$, we have

$$
c_{1}=-2 z_{1}^{(1)}, \quad c_{2}=-2 z_{1}^{(2)}
$$

Moreover, we can construct the relationship (22) between the following coordinates:

$$
\begin{gathered}
y_{1}^{(1)}=x_{1}, \quad y_{2}^{(1)}=-2 x_{1}^{2}+2 x_{2}, \quad z_{1}^{(1)}=x_{1}, \\
z_{2}^{(1)}=-2 x_{1}^{2}+2 x_{2}-2 x_{1}, \\
y_{1}^{(2)}=x_{2}, \quad y_{2}^{(2)}=x_{1}-x_{2}, \quad z_{1}^{(2)}=x_{2}, \\
z_{2}^{(2)}=x_{1}-3 x_{2},
\end{gathered}
$$

which implies the following diffeomorphism between $Z_{2}^{(1)}$ and $Z_{2}^{(2)}$ :

$$
\begin{aligned}
z_{1}^{(1)}= & 3 z_{1}^{(2)}+z_{2}^{(2)}, \\
z_{2}^{(1)}= & -18\left(z_{1}^{(2)}\right)^{2}-2\left(z_{2}^{(2)}\right)^{2} \\
& -12 z_{1}^{(2)} z_{2}^{(2)}-4 z_{1}^{(2)}-2 z_{2}^{(2)}, \\
z_{1}^{(2)}= & \left(z_{1}^{(1)}\right)^{2}+z_{1}^{(1)}+\frac{1}{2} z_{2}^{(1)}, \\
z_{2}^{(2)}= & -3\left(z_{1}^{(1)}\right)^{2}-2 z_{1}^{(1)}-\frac{3}{2} z_{2}^{(1)} .
\end{aligned}
$$

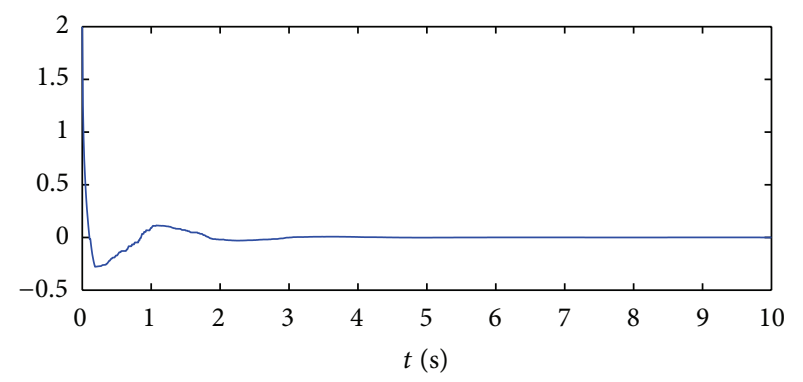

- Output of $y_{2}^{(i)}$

FIGURE 3: Trajectories of state $y_{2}^{(i)}$.

Now, we discuss the coupled terms

$$
\begin{aligned}
\widehat{V}\left(Z_{n}^{(1)}, 1\right)= & \frac{1}{2\left(z_{1}^{(1)}\right)^{2}}+\frac{1}{2\left(z_{2}^{(1)}\right)^{2}} \\
\widehat{V}\left(Z_{n}^{(1)}, 2\right)= & \frac{1}{2}\left(\left(z_{1}^{(1)}\right)^{2}+z_{1}^{(1)}+\frac{1}{2} z_{2}^{(1)}\right)^{2} \\
& +\frac{1}{2}\left(-3\left(z_{1}^{(1)}\right)^{2}-2 z_{1}^{(1)}-\frac{3}{2} z_{2}^{(1)}\right)^{2}, \\
\widehat{V}\left(Z_{n}^{(2)}, 1\right)= & \frac{1}{2}\left(3 z_{1}^{(2)}+z_{2}^{(2)}\right)^{2} \\
& +\frac{1}{2}\left(-18\left(z_{1}^{(2)}\right)^{2}-2\left(z_{2}^{(2)}\right)^{2}\right. \\
\widehat{V}\left(Z_{n}^{(2)}, 2\right)= & \frac{1}{2}\left(z_{1}^{(2)}\right)^{2}+\frac{1}{2}\left(z_{2}^{(2)}\right)^{2} .
\end{aligned}
$$

From (34), we have that

$$
\begin{aligned}
\Delta \widehat{V}_{1}\left(Z_{1}^{(1)}, 1\right)= & \frac{1}{2} z_{1}^{(1)}, \quad \Delta \widehat{V}_{2}\left(Z_{2}^{(1)}, 1\right)=\frac{1}{2} z_{2}^{(1)}, \\
\Delta \widehat{V}_{1}\left(Z_{1}^{(1)}, 2\right)= & 5\left(z_{1}^{(1)}\right)^{3}+7\left(z_{1}^{(1)}\right)^{2}+\frac{5}{2} z_{1}^{(1)} \\
\Delta \widehat{V}_{2}\left(Z_{2}^{(1)}, 2\right)= & 5\left(z_{1}^{(1)}\right)^{2}+2 z_{1}^{(1)}+\frac{5}{4} z_{2}^{(2)}, \\
\Delta \widehat{V}_{1}\left(Z_{1}^{(2)}, 1\right)= & 162\left(z_{1}^{(2)}\right)^{3}+72\left(z_{1}^{(2)}\right)^{2}+\frac{25}{2} z_{1}^{(2)}, \\
\Delta \widehat{V}_{2}\left(Z_{2}^{(2)}, 1\right)= & 216\left(z_{1}^{(2)}\right)^{3}+2\left(z_{2}^{(2)}\right)^{3} \\
& +108\left(z_{1}^{(2)}\right)^{2} z_{2}^{(2)}+24 z_{1}^{(2)}\left(z_{2}^{(2)}\right)^{2}+84\left(z_{1}^{(2)}\right)^{2} \\
& +4\left(z_{2}^{(2)}\right)^{2}+32 z_{1}^{(2)} z_{2}^{(2)}+11 z_{1}^{(2)}+\frac{5}{2} z_{2}^{(2)}, \\
\Delta \widehat{V}_{1}\left(Z_{1}^{(2)}, 2\right)= & \frac{1}{2} z_{1}^{(2)}, \quad \Delta \widehat{V}_{2}\left(Z_{2}^{(2)}, 2\right)=\frac{1}{2} z_{2}^{(2)} .
\end{aligned}
$$


Then, from (40), we can construct the control law $v^{*}$ as follows:

$$
\begin{aligned}
v_{1}= & -z_{1}^{(1)}+\frac{\partial c_{1}^{(1)}}{\partial z_{1}^{(1)}} F_{1}^{(1)}-\lambda_{11} \Delta \widehat{V}_{2}\left(Z_{n}^{(1)}, 1\right)-\lambda_{12} \Delta \widehat{V}_{2}\left(Z_{n}^{(1)}, 2\right) \\
= & -5\left(z_{1}^{(1)}\right)^{2}+z_{1}^{(1)}-\frac{1}{4} z_{2}^{(1)}, \\
v_{2}= & -z_{1}^{(2)}+\frac{\partial c_{1}^{(2)}}{\partial z_{1}^{(2)}} F_{1}^{(2)}-\lambda_{21} \Delta \widehat{V}_{2}\left(Z_{n}^{(2)}, 1\right)-\lambda_{22} \Delta \widehat{V}_{2}\left(Z_{n}^{(2)}, 2\right) \\
= & -432\left(z_{1}^{(2)}\right)^{3}-4\left(z_{2}^{(2)}\right)^{3}-216\left(z_{1}^{(2)}\right)^{2} z_{2}^{(2)}-48 z_{1}^{(2)}\left(z_{2}^{(2)}\right)^{2} \\
& -168\left(z_{1}^{(2)}\right)^{2}-8\left(z_{2}^{(2)}\right)^{2}-64 z_{1}^{(2)} z_{2}^{(2)}-19 z_{1}^{(2)}-6 z_{2}^{(2)} .
\end{aligned}
$$

Moreover, by (9), we can design the original control $u$ as follows:

$$
\begin{aligned}
u_{1}^{*}= & -4 x_{1}^{3}-\frac{9}{4} x_{1}^{2}+4 x_{1} x_{2}+\frac{3}{4} x_{1}-\frac{5}{4} x_{2} \\
u_{2}^{*}= & -4 x_{1}^{3}-108 x_{2}^{3}-12 x_{1}^{2} x_{2}-36 x_{1} x_{2}^{2} \\
& -8 x_{1}^{2}-49 x_{2}^{2}-16 x_{1} x_{2}-5 x_{1}-2 x_{2}
\end{aligned}
$$

which guarantee the asymptotically stability of the closedloop system.

Given initial values $x_{1}(0)=2$ and $x_{2}(0)=0$, the simulation results of the above discussion are presented as follows. Figure 1 shows the states of Markovian switchings. Correspondingly, the trajectories of $y_{1}^{(i)}$ and $y_{2}^{(i)}$ are shown in Figures 2-3, respectively. Obviously, the control law $v^{*}$ asymptotically stabilizes the closed-loop system.

\section{Conclusion}

This paper has discussed the linearization and stabilization problems for a class of general Markovian jump nonlinear systems. Following with the Markovian switchings, the appropriate coordinate is adopted under which the system can be transformed into the canonical form corresponding to the relative degree set. Then, the backstepping technique is applied in the stabilizing control design of Markovian jump nonlinear systems. Further efforts could be concentrated on the study of the linearization and stabilization of Markovian jump nonlinear systems with arbitrary relative degree set $\left\{\rho_{1}, \rho_{2}, \ldots, \rho_{n}\right\}$.

\section{Acknowledgment}

This work is partially supported by the National Basic Research Program of China (973 Program) (Grant no. 2012CB215203), the National Natural Science Foundation of China (no. 61203043 and no. 51036002) and the Fundamental Research Funds for the Central Universities.

\section{References}

[1] M. M. Mahmoud, J. Jiang, and Y. Zhang, Active Fault Tolerant Control Systems, vol. 287 of Lecture Notes in Control and Information Sciences, Springer, New York, NY, USA, 2003.

[2] M. Mariton, Jump Linear Systems in Automatic Control, Marcel Dekker, New York, NY, USA, 1990.

[3] R. C. Tsaur, "A fuzzy time series-Markov chain model with an application to forecast the exchange rate between the Taiwan and US dollar," International Journal of Innovative Computing, Information and Control, vol. 8, no. 7, pp. 4931-4942, 2012.

[4] F. Kojima and J. S. Knopp, "Inverse problem for electromagnetic propagation in a dielectric medium using Markov chainMonte Carlo method," International Journal of Innovative Computing, Information and Control, vol. 8, no. 3, pp. 2339-2346, 2012.

[5] Q. Ahmed, A. Iqbal, I. Taj, and K. Ahmed, "Gasoline engine intake manifold leakage diagnosis/prognosis using hidden Markov model," International Journal of Innovative Computing, Information and Control, vol. 8, no. 7, pp. 4661-4674, 2012.

[6] P. Shi, E.-K. Boukas, and R. K. Agarwal, "Kalman filtering for continuous-time uncertain systems with Markovian jumping parameters," IEEE Transactions on Automatic Control, vol. 44, no. 8, pp. 1592-1597, 1999.

[7] P. Shi, E.-K. Boukas, and R. K. Agarwal, "Control of Markovian jump discrete-time systems with norm bounded uncertainty and unknown delay," IEEE Transactions on Automatic Control, vol. 44, no. 11, pp. 2139-2144, 1999.

[8] Z. Wang, H. Qiao, and K. J. Burnham, "On stabilization of bilinear uncertain time-delay stochastic systems with Markovian jumping parameters," IEEE Transactions on Automatic Control, vol. 47, no. 4, pp. 640-646, 2002.

[9] J. Lam, Z. Shu, S. Xu, and E.-K. Boukas, "Robust $H_{\infty}$ control of descriptor discrete-time Markovian jump systems," International Journal of Control, vol. 80, no. 3, pp. 374-385, 2007.

[10] L. Wu, X. Su, and P. Shi, "Sliding mode control with bounded $L_{2}$ gain performance of Markovian jump singular time-delay systems," Automatica, vol. 48, no. 8, pp. 1929-1933, 2012.

[11] L. Wu, P. Shi, and H. Gao, "State estimation and slidingmode control of Markovian jump singular systems," IEEE Transactions on Automatic Control, vol. 55, no. 5, pp. 1213-1219, 2010.

[12] X. Su, P. Shi, L. Wu, and Y. Song, "A novel approach to filter design for T-S fuzzy discrete-time systems with time-varying delay," IEEE Transactions on Fuzzy Systems, vol. 20, no. 6, pp. 1114-1129, 2012.

[13] R. Yang, H. Gao, and P. Shi, "Novel robust stability criteria for stochastic Hopfield neural networks with time delays," IEEE Transactions on Systems, Man, and Cybernetics B, vol. 39, no. 2, pp. 467-474, 2009.

[14] R. Yang, P. Shi, G.-P. Liu, and H. Gao, "Network-based feedback control for systems with mixed delays based on quantization and dropout compensation," Automatica, vol. 47, no. 12, pp. 2805-2809, 2011.

[15] Z. Fei, H. Gao, and P. Shi, "New results on stabilization of Markovian jump systems with time delay," Automatica, vol. 45, no. 10, pp. 2300-2306, 2009.

[16] X. Mao and C. Yuan, Stochastic differential equations with Markovian switching, Imperial College Press, London, UK, 2006.

[17] M. D. S. Aliyu, "Passivity and stability of nonlinear systems with Markovian jump parameters," in Proceedings of the American Control Conference (ACC '99), pp. 953-957, June 1999. 
[18] Z. W. Lin, Y. Lin, and W. H. Zhang, "A unified design for state and output feedback $H_{\infty}$ control of nonlinear stochastic Markovian jump systems with state and disturbance-dependent noise," Automatica, vol. 45, no. 12, pp. 2955-2962, 2009.

[19] Z. W. Lin, J. Z. Liu, W. H. Zhang, and Y. G. Niu, "Stabilization of interconnected nonlinear stochastic Markovian jump systems via dissipativity approach," Automatica, vol. 47, no. 12, pp. 27962800, 2011.

[20] Z.-J. Wu, X.-J. Xie, P. Shi, and Y.-Q. Xia, "Backstepping controller design for a class of stochastic nonlinear systems with Markovian switching," Automatica, vol. 45, no. 4, pp. 997-1004, 2009.

[21] J. C. Willems, "Dissipative dynamical systems. I. General theory," Archive for Rational Mechanics and Analysis, vol. 45, pp. 321-393, 1972.

[22] D. J. Hill and P. J. Moylan, "The stability of nonlinear dissipative systems," IEEE Transactions on Automatic Control, vol. 21, no. 5, pp. 708-711, 1976.

[23] C. I. Byrnes, A. Isidori, and J. C. Willems, "Passivity, feedback equivalence, and the global stabilization of minimum phase nonlinear systems," IEEE Transactions on Automatic Control, vol. 36, no. 11, pp. 1228-1240, 1991.

[24] W. Lin and T. Shen, "Robust passivity and feedback design for minimum-phase nonlinear systems with structural uncertainty," Automatica, vol. 35, no. 1, pp. 35-47, 1999.

[25] A. Isidori, Nonlinear Control System, Springer, Berlin, Germany, 1994.

[26] H. K. Khalil, Nonlinear Systems, Prentice Hall, New York, NY, USA, 2002. 


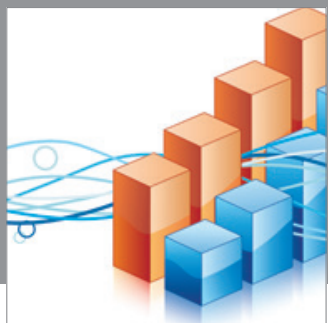

Advances in

Operations Research

mansans

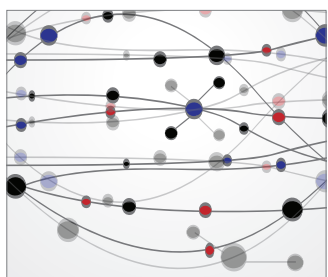

The Scientific World Journal
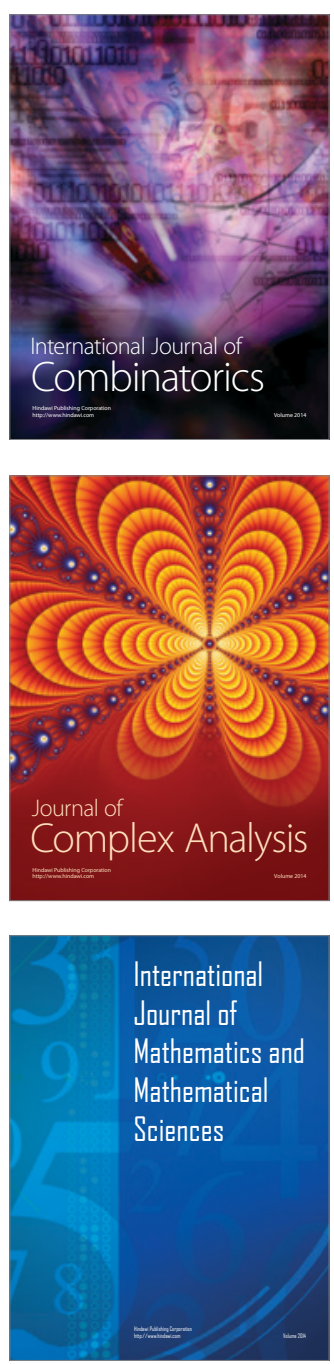
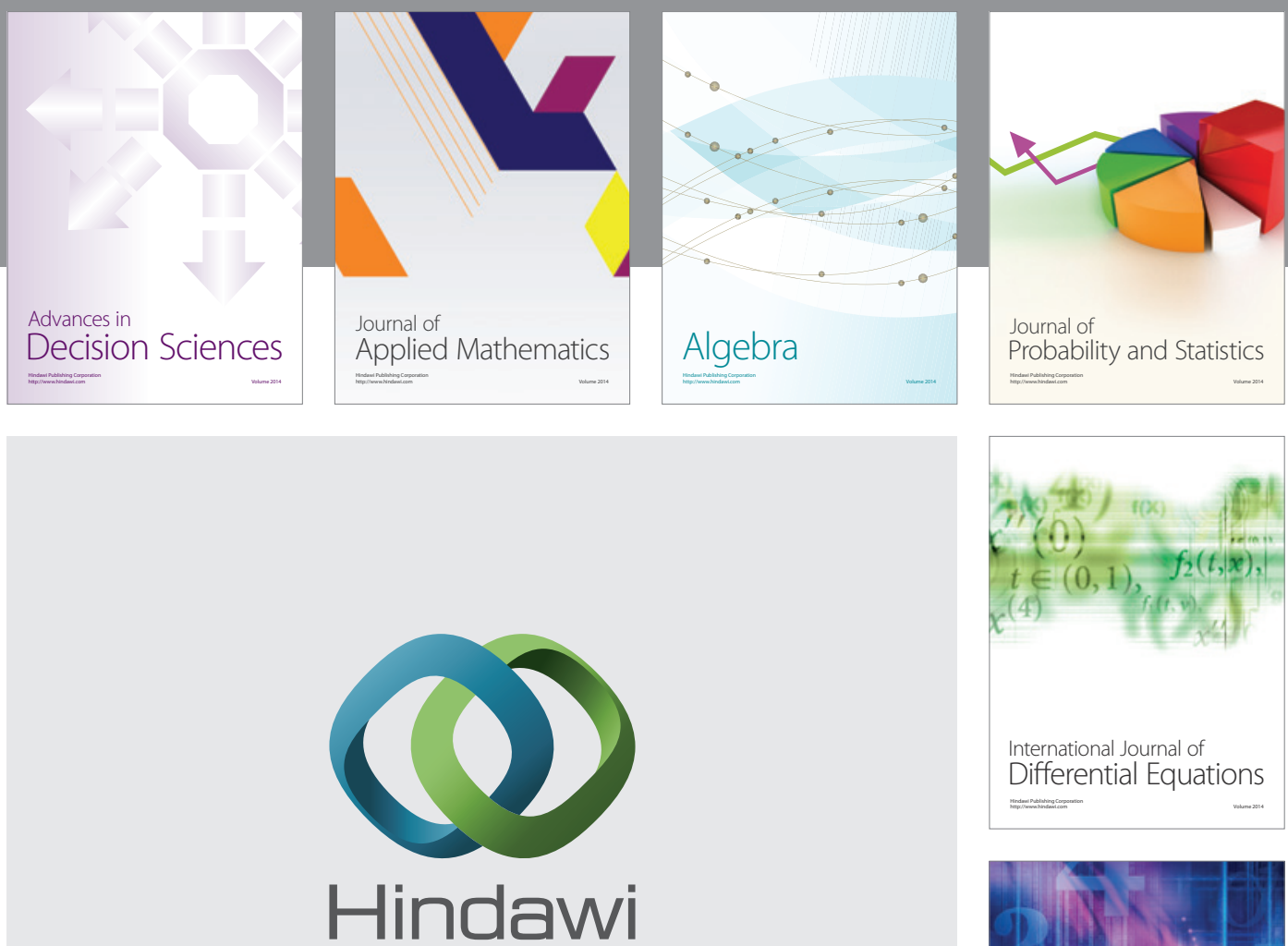

Submit your manuscripts at http://www.hindawi.com
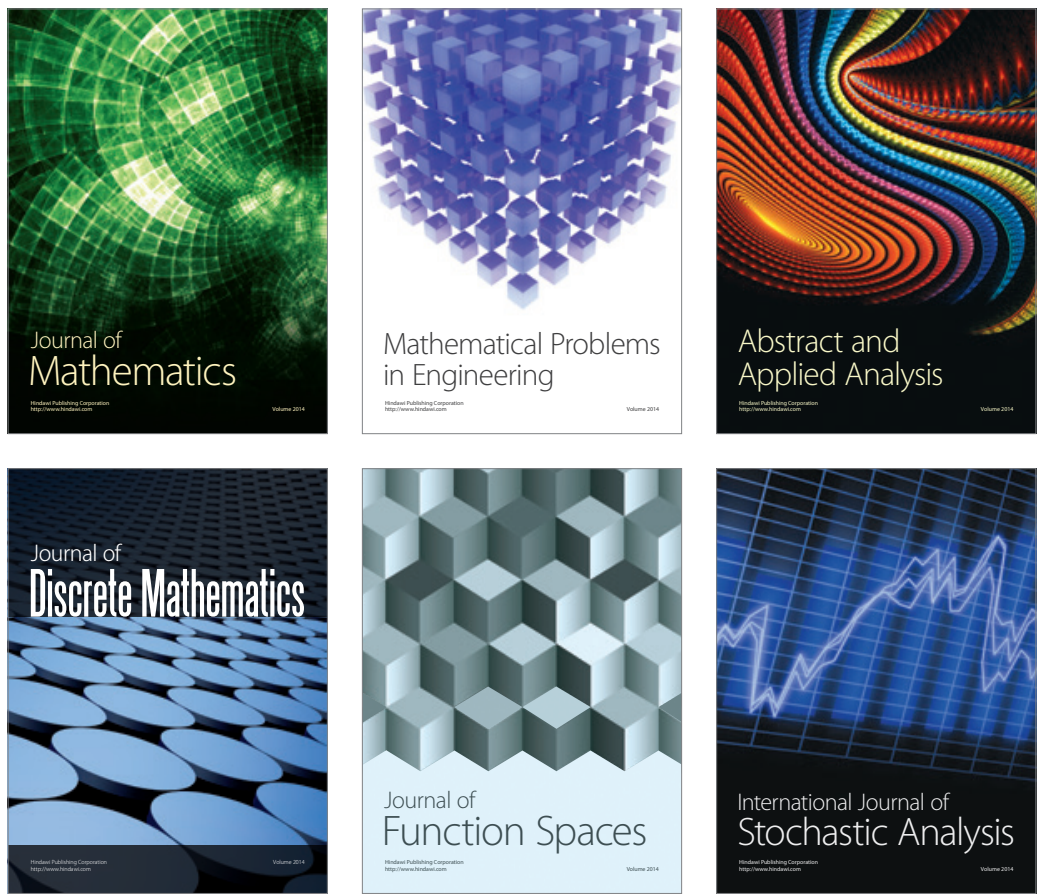

Journal of

Function Spaces

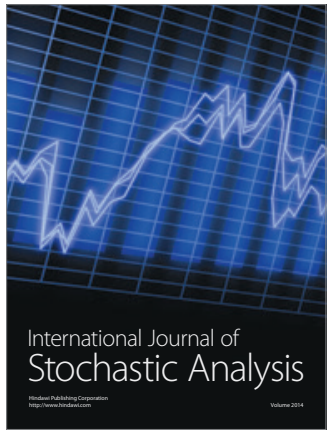

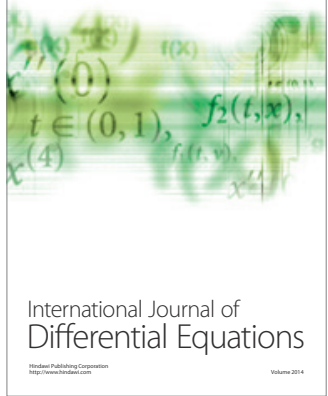
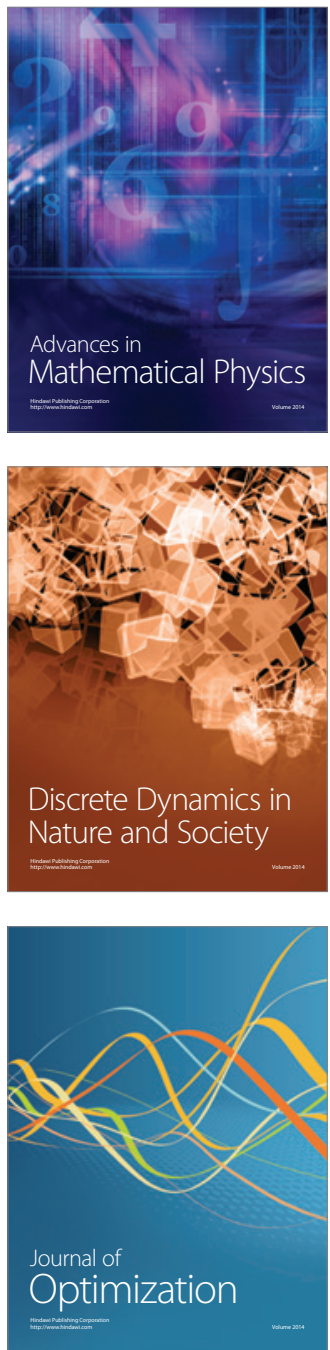